\title{
Gabinetes de comunicación del Sistema de Ciencia, Tecnología e Innovación: el puente que cruza la I+D+i para llegar a los medios
}

\author{
Department of Communication of the Science, \\ Technology and Innovation System: the bridge \\ that crosses I + D + i to reach the media
}

\author{
AUTORA \\ Ana Bellón Rodríguez \\ http://orcid.org/0000-0002-8779-1110 \\ CSIC and USC
}

Fuentes de financiación "Usos y preferencias informativas en el nuevo mapa de medios en España: modelos de periodismo para dispositivos móviles" (Referencia: CSO2015-64662-C4-4-R), del Ministerio de Economía, Industria y Competitividad. Este proyecto está cofinanciado por el fondo estructural FEDER. También se enmarca en la Red XESCOM.

\section{Resumen}

Este artículo, de carácter exploratorio-descriptivo, pone el foco en un elemento clave en la difusión periodística de la labor de los agentes de ejecución del Sistema Español de Ciencia, Tecnología e Innovación, tales como universidades y organismos públicos: los gabinetes de comunicación. Éstos han emergido en las últimas décadas y generan información científica y tecnológica susceptible de ser objeto de cobertura mediática. Formula preguntas y ofrece respuestas sobre este tipo de gabinetes en el ámbito de la comunicación externa aplicando para ello la regla de las 6 ws de la profesión periodística. Establece sus particularidades y singularidades en aspectos como los valores noticia, las notas de prensa o las ruedas de prensa, que se explican por el tipo de información con la que trabajan, la especializada $y$, dentro de ella, la científica y tecnológica. Analiza su relación con dos elementos implicados a su vez en los contenidos que difunden a medios: el personal investigador que genera el tema y

\section{Abstract}

This article, an exploratory-descriptive study, focus on a key element in the journalistic diffusion of the work of the executing agents of the Spanish System of Science, Technology and Innovation, such as universities and public organizations: the communication departments. These have emerged in recent decades and generate scientific and technological information likely to be the subject of media coverage. It formulates questions and offers answers on this type of departements in the field of the external communication with the rule of the 6 ws of the journalistic profession. It establishes its particularities and singularities in aspects such as news values, press releases or press conferences, which are explained by the type of information with which they work, specialized and, within it, scientific and technological. The article analyzes their relationship with two elements involved in the content that disseminate to the media: the research staff that generates the subjetc and the journalist who receives and prepares 
el periodista que lo recibe y prepara para el medio. Propone buenas prácticas a seguir en su papel de intermediador entre ambos, en las que debe primar el respeto profesional y la compresión de las diferencias y similitudes entre el mundo científico y el periodístico.

Palabras clave: Gabinete; Comunicación; Agente; Ciencia; Tecnología; Innovación it for the media. The article proposes good practices to follow in his role as intermediary between both, in which professional respect and understanding of the differences and similarities between the scientific and journalistic world must prevail.

Key words: Department; Communication; Agent; Science; Technology; Innovation

\section{Introducción, Objetivos y Metodología}

La Ley 14/2011, de 1 de junio, de la Ciencia, la Tecnología y la Innovación establece el marco para el fomento de la investigación científica y técnica y sus instrumentos de coordinación general. Su fin es contribuir a la generación, difusión y transferencia del conocimiento para resolver los problemas esenciales de la sociedad.

Entre sus medidas para "una ciencia del siglo XXI" figura el compromiso con la difusión universal del conocimiento científico y tecnológico. Reconoce las actividades de divulgación y cultura científica y tecnológica como consustanciales a la carrera investigadora y entre los deberes que fija para el personal investigador figuran difundir los resultados de sus investigaciones (incluye un artículo dedicado a la difusión en acceso abierto) y procurar que su labor sea relevante para la sociedad.

En su articulado se alude al Sistema Español de Ciencia, Tecnología e Innovación. Éste incluye a agentes, públicos y privados, con tres funciones: financiación, ejecución o coordinación. Este artículo se centra en los de ejecución, entidades públicas y privadas que realizan o dan soporte a la investigación científica y técnica e innovación. Su labor puede entrar en la agenda mediática y ser objeto de cobertura por parte de los medios de comunicación.

Y es que la ciencia, la tecnología y la innovación son productos que pueden ser consumidos a través de los medios, siempre y cuando se ofrezcan en tono divulgativo y con estilo periodístico. Son noticia (FECYT, 2016). Pero, ¿cómo llegan, por lo general, los resultados de investigación, las iniciativas de divulgación y otras líneas de actuación de los agentes del Sistema con funciones de ejecución a los medios de comunicación? La respuesta a esta pregunta se encuentra, fundamentalmente, en los gabinetes de comunicación.

Una definición genérica los presenta como "fuentes activas, organizadas y habitualmente estables de información que cubren las necesidades comunicativas tanto internas como externas de aquellas organizaciones y/o personas de relieve que desean transmitir de sí mismas una imagen positiva a la sociedad influyendo de esta forma en la opinión pública" (Ramírez, 1995: 27). Fernández y Torre (2009: 21-30) apuntan que "son unidades dirigidas en general por profesionales de la comunicación y en particular por periodistas", mientras García (2009: 12) incide en que "se encargan de la planificación, implementación y evaluación de la política comunicativa de la organización". 
Surgieron en España en los albores de la transición política bajo el manto protector de las instituciones oficiales. Después los incorporaron los partidos políticos y paulatinamente el resto de grupos sociales con relativa presencia pública (Ramírez, 1995: 19). Martín (1999: 80) distingue ocho grandes tipos: de instituciones públicas, organizaciones internacionales, cámaras del comercio, partidos políticos, embajadas, universidades o centros educativos, confederaciones empresariales y empresas privadas, e incide en que "todos tienen alguna característica concreta que los diferencia de los demás, aunque todos realicen funciones similares comunicativas".

En este contexto, se propone incluir en esa tipología a los gabinetes de comunicación de los agentes de ejecución del Sistema Español de Ciencia, Tecnología e Innovación, en el cual se integrarían los de universidades, tanto públicas como privadas, los de los ocho organismos públicos de investigación de la Administración General del Estado, los de organismos de investigación de Comunidades Autónomas, los de parques científicos y tecnológicos y cualquier entidad que se ajuste a la definición propuesta en la Ley 14/2011 para agentes de ejecución. No se dispone de ningún estudio cuantitativo acerca de cuántos de estos agentes cuentan con gabinete de comunicación y en algunos casos esta labor puede estar canalizada a través de agencias de comunicación. Pero, en general, y en base a nuestro conocimiento del tema, se puede afirmar que la mayoría han ido implantando en las últimas décadas este elemento en su organigrama.

Las definiciones generales de gabinetes de comunicación son extrapolables a los agentes de ejecución del Sistema, incluyendo una importante particularidad: desarrollan su labor no sólo en el ámbito de la comunicación corporativa, sino también en el de la especializada. A su vez, dentro de la especializada se dedican al periodismo científico y dentro del mismo a una o varias áreas: Ciencias Marinas, Ciencias Agrarias, Humanidades y Ciencias Sociales...

Capriotti (1999) define la comunicación corporativa como "la totalidad de los recursos de comunicación de los que dispone una organización para llegar efectivamente a sus públicos". En cuanto a la especialización periodística, su auge se enmarca en la necesidad de una interpretación adecuada de la naturaleza científica y tecnológica del progreso (Bell, 1973) y responde a varias crisis (Fernández del Moral, 1993: 24). Dentro de ella, el periodismo científico aborda la información que procede de los avances, intereses o hechos suministrados por la ciencia (Elías, 2008: 30).

En el estado de la cuestión sobre los gabinetes de comunicación en España figuran estudios sobre sus funciones en la administración local (Camacho, 2001), la estructura funcional del gabinete de prensa político en los entornos informativos locales (Mateos, 2003), en los hospitales públicos de Galicia (Costa, 2011), su presencia en algunas Comunidades Autónomas (Almansa, 2006) o su historia (Almansa, 2004). También ha prestado atención al paso del gabinete de prensa al de comunicación (2011). Sin embargo, hasta donde nuestro conocimiento alcanza, no se dispone de estudios específicos sobre los gabinetes de comunicación de los agentes del Sistema de I+D+i.

En este contexto, se ha realizado una labor de búsqueda y consulta bibliográfica de obras de referencia en el panorama nacional sobre comunicación corporativa, institucional, gabinetes 
de comunicación, periodismo especializado y periodismo científico. Ello ha permitido ir de lo general a lo particular, caracterizando y ofreciendo respuestas específicas a las principales cuestiones relacionadas con los gabinetes de comunicación de I+D+i y proponiendo buenas prácticas en sus relaciones con el personal investigador de la entidad para la que prestan sus servicios y con los periodistas de los medios de comunicación a los que dirigen sus acciones.

El objetivo es ofrecer una visión general, de tipo aproximativo, sobre los gabinetes de comunicación especializados en I+D+i que desarrollan su labor para agentes de ejecución del Sistema Español de Ciencia, Tecnología e Innovación.

\section{Resultados}

\section{¿Qué comunicar y cuándo?}

Las empresas e instituciones son estructuras comunicativas que abarcan todos sus procesos y actividades (AA.VV, 2002). Son, por tanto, portadoras de información, tanto general como especializada. Dentro de esta última, se sitúa la relacionada con la investigación científica y tecnológica, desarrollo e innovación.

Las siglas $\mathrm{I}+\mathrm{D}+\mathrm{i}$ han irrumpido en los medios, pero, ¿es consciente el público general de a qué nos referimos cuando hablamos de investigación, de innovación o de tecnología? Es decir, ¿qué estamos comunicando? En una de las normas UNE, la 166000, se definen estos conceptos.

La investigación es una indagación original y planificada que persigue descubrir nuevos conocimientos y una superior comprensión en el ámbito científico y tecnológico, la innovación es una actividad cuyo resultado es la obtención de nuevos productos o procesos o mejoras sustancialmente significativas en los ya existentes y la tecnología es un conjunto de recursos técnicos propios de una actividad que pueden ser utilizados de forma sistemática para el diseño, desarrollo, fabricación y comercialización de productos o para la prestación de servicios (AENOR, 2008).

Una de las principales líneas de actuación de los gabinetes de los agentes de ejecución del Sistema Español de Ciencia, Tecnología e Innovación está vinculada a los proyectos de I+D+i, especialmente si son financiados por convocatorias públicas competitivas de programas del Plan Estatal de Investigación Desarrollo e Innovación o del Programa Marco H2020. Dentro de los proyectos se puede distinguir entre investigación, desarrollo tecnológico, demostración tecnológica e innovación tecnológica (Dirección Xeral de Investigación e Desenvolvemento, 2003).

Otras líneas de actuación con potencial impacto mediático son las publicaciones científicas, especialmente si están publicadas en revistas de impacto indexadas en las principales bases de datos, como SCOPUS; las actividades de divulgación científica promovidas por la entidad, sobre todo durante eventos como la Semana de la Ciencia o la Noche de los Investigadores; resultados de tesis doctorales realizadas en la institución; avances en el ámbito de la transferencia de tecnología (patentes, modelos de utilidad, variedades vegetales...); contratos con empresas, de especial relevancia en el marco de la necesidad de incrementar la financiación privada en $\mathrm{I}+\mathrm{D}+\mathrm{i}$; convenios y acuerdos con otras entidades, tanto públicas como privadas; 
premios y distinciones a la entidad o a su personal; iniciativas de interés general; posición en rankings de reconocido prestigio...

A todo ello se une su labor en el ámbito de la comunicación institucional relacionada, por ejemplo, con nombramientos y relevos en los órganos de dirección.

Hay, por tanto, una amplia variedad de temas que pueden ser objeto de difusión desde el gabinete de I+D+i. Para decidir si son o no susceptibles de difundir a medios hay que tener en cuenta las leyes de la noticiabilidad, entendida por Ramírez (1995: 39) como "un conjunto de elementos que intervienen en la definición del hecho noticioso". La persona que esté al frente a un gabinete de un agente de ejecución del Sistema Español de Ciencia, Tecnología e Innovación tendrá que valorar si el hecho cumple o no los criterios de noticiabilidad. Según Martínez (1983: 40), una noticia es "un hecho verdadero, inédito y actual que se comunica a un público que se considera masivo una vez que ha sido recogido, interpretado y valorado por los sujetos promotores que controlan el medio para su difusión". Según Fontcuberta (2011) "el tiempo es el elemento básico para distinguir la noticia de otras informaciones. El acontecimiento periodístico tiene como base de su existencia la actualidad: cuanto más inmediata, mejor".

Partiendo de Galtung y Ruge (1973) e incorporando diversas aportaciones de la periodística, Túñez (1999: 95) enumera los valores noticia a los que se otorga mayor peso en el proceso de selección: actualidad, cantidad de personas implicadas en el acontecimiento, frecuencia, grado de nivel jerárquico de los sujetos implicados, novedad, proximidad, proyección, consecuencias y relevancia. Si un hecho reúne los denominados valores noticia puede valorarse desde el gabinete su difusión a medios y establecer la estrategia a seguir. Si no los cumple, el gabinete puede proponer otras vías para dar una cierta visibilidad al hecho: newsletter, página web institucional, redes sociales...

Así, los valores noticia entran de lleno en el gatekeeping, entendido como un proceso que hace posible que de todos los millones de hechos noticiosos que se producen a diario en el mundo los medios sólo elijan un puñado (Ramírez, 1995: 40). Ello ha sido abordado por Shoemaker (1991: 10), quien lo explica así: las fuentes informativas envían numerosos mensajes informativos a los gatekeeper que operan en los medios. Éstos llevan a cabo un proceso de selección (gatekeeping) de todos ellos. En base a ello, unos ítems son rechazados y quedan fuera, sin traspasar el umbral de medio informativo, mientras que otros son escogidos.

En este sentido, las informaciones generadas por los agentes de ejecución del Sistema de Ciencia, Tecnología e Innovación pasan un doble proceso de selección:

Primero, el del propio gabinete, que decidirá si se difunde o no aplicando los valores noticias y teniendo en cuenta además la política de comunicación la cual, según Carrascosa (1992: 23), debe cumplir tres requisitos: depender del máximo órgano dentro de la organización, partir de un enfoque global de la comunicación y otorgar a la comunicación interna un carácter primordial.

Después, el del propio medio. Así, la nota de prensa elaborada y enviada a los medios desde el gabinete puede o no tener repercusión en los medios y ésta puede ser mayor o menor. En 
el medio se seleccionan las informaciones que reciben de nuevo en base a los valores noticia y a otro cúmulo de factores: espacio del que disponga, informaciones similares cubiertas recientemente, interés para su público, coincidencia en el día de envío de la información de un hecho de especial relevancia que requiera prácticamente toda la atención del medio... Por tanto, a partir de la selección hecha por el gatekeeper se realiza una nueva selección para hacer la jerarquización y para decidir cuáles son los asuntos que formarán parte de la portada del medio (Túñez, 1999: 86). Rositi (1980) diferencia tres niveles de selección: la reglamentación del derecho de acceso al circuito informativo, la jerarquización de las noticias y la tematización.

El momento en el que se comunica una información es de nuevo otra decisión que corresponde adoptar al responsable del gabinete, en consenso con el personal investigador que genera el tema, teniendo presente, siempre, la importancia de la actualidad y la premisa de evitar filtraciones. Sobre esto último, es importante que los periodistas que trabajan en medios respeten la dinámica del gabinete de comunicación del agente de ejecución del Sistema.

Además, el responsable del gabinete deberá intentar combinar tanto las necesidades y oportunidades comunicativas de la entidad con una actividad que no sature a los medios de informaciones del mismo tipo y, si trabaja para un agente con diferentes áreas y líneas de investigación, es interesante que intente dar visibilidad, periódicamente, a todas ellas. Con ello reforzará mediáticamente la labor multidisciplinar del agente.

Los cambios en el periodismo han sido notables en las últimas décadas. No obstante, se siguen manteniendo unos principios para que las informaciones sean calificadas como tales (La Colic, 2006), exigencias inexcusables y que tienen que estar presentes en todas las informaciones: veracidad, actualidad, novedad e inmediatez, interés público o relevancia (Cebrián, 2012: 56). Por ello, estos criterios deben guiar siempre la labor del responsable del gabinete de comunicación de I+D+i.

\section{¿Quién comunica desde un gabinete de comunicación de un agente de ejecución del Sistema?}

Los directores de comunicación que trabajan en un gabinete son habitualmente periodistas que conocen a la perfección todo lo concerniente al proceso de selección, producción y codificación de la noticia (Ramírez, 1995: 39). Ello redunda en el éxito de las acciones de comunicación de la entidad. Son profesionales de la comunicación y, por tanto, están capacitados para preparar textos periodísticos y determinar lo que puede y no ser noticia. Conocen, además, las necesidades y los ritmos de trabajo, diferentes y específicos, en la prensa, la radio, la televisión e Internet. Todo ello tienen que hacérselo ver al personal que genera la información, pues pueden producirse situaciones en las que, por desconocimiento de los medios, no se comprenda por qué algo no es objeto de difusión periodística o por qué debe titularse la nota de prensa de una manera y no de otra. Éstos son solo algunos ejemplos de discrepancias entre científicos y periodistas debidas, fundamentalmente, a las diferencias entre ambos mundos.

El responsable de un gabinete de comunicación tiene que disponer de un mailing completo y en permanente actualización de los medios a los que dirige sus acciones, tanto en el ámbito local como autonómico, nacional e internacional. En función del tipo de información tendrá que decidir a qué ámbito dirige el tema o dónde tiene que reforzarlo. Además, es importante 
que al tratase de temas científicos tenga localizados y mantenga contactos con los medios de comunicación especializados tanto en la I+D+i en general como en áreas específicas.

Es fundamental que mantenga una relación fluida, profesional, estable y basada en la confianza y el respeto mutuo con los dos colectivos que forman parte de su día a día: el personal investigador y los periodistas.

Si bien debe seguir de cerca la actualidad científica de la entidad para la que desarrolla su labor (concesiones de proyectos, publicaciones...), es oportuno que se establezca un protocolo por el cual se le mantendrá informado permanentemente de cualquier hecho que pueda ser objeto de difusión mediática y que en dicho protocolo se indique que cualquier llamada o consulta de un medio de comunicación por iniciativa propia deberá derivarse al gabinete, desde donde se realizará la gestión. Por tanto, la comunicación interna es fundamental. Está integrada en la estrategia general de comunicación de las empresas e instituciones (Andrade, 2004) y de su buena organización y funcionamiento dependerá en gran medida la comunicación externa (Elías, 1998).

A él le corresponde aplicar los valores noticia a las informaciones que genera el personal investigador, definido en la Ley 14/2011 como el que "estando en posesión de la titulación exigida en cada caso lleva a cabo una actividad investigadora, entendida como el trabajo creativo realizado de forma sistemática para incrementar el volumen de conocimientos, incluidos los relativos al ser humano, la cultura y la sociedad, el uso de esos conocimientos para crear nuevas aplicaciones, su transferencia y su divulgación", para decidir, en base a criterios objetivos y profesionales, si es o no objeto de difusión a medios.

Su principal tarea es, por tanto, la selección de informaciones que pueden ser susceptibles de comunicación a medios y la posterior preparación de las mismas. Mientras, la principal tarea del personal investigador en el ámbito de la comunicación periodística es mantener informado al gabinete de los hechos que puedan ser objeto de cobertura mediática o que desee hacer llegar a la sociedad a través de los medios y colaborar con el gabinete en lo que se le requiere al respecto: información, disponibilidad para atender a los medios... Ambos deben respetar sus parcelas de trabajo y actuar en común para un mismo fin: dar visibilidad a la entidad en los medios de comunicación.

Cuando el responsable del gabinete se dirija a los medios de comunicación a través de diferentes acciones debe tener presente, tal y como afirma Cebrián (2012: 57), que "la información empresarial e institucional exige los mismos principios que cualquier otra modalidad de periodismo. No existen dos clases de periodismo, sino el mismo examinado desde diversas perspectivas". A ello se une que ni en un caso ni en otro está permitida la mentira ni el engaño. En ambos casos la información tiene que ser veraz (Moreno, 2009).

\section{¿Dónde comunica un gabinete de comunicación de un agente de ejecu- ción del Sistema?}

Tal y como apunta Cebrián (2012: 17) "el periodismo en las empresas e instituciones no debe contemplarse como una excepción aparte. Se enmarca en las estrategias de comunicación y comparte los objetivos y fines de cada corporación". 
Las funciones de un gabinete de comunicación se desarrollan en dos grandes ámbitos: comunicación interna y externa. Este artículo se centra en el segundo ámbito de actuación y dentro del mismo en los medios de comunicación: prensa, radio, televisión e Internet (sobre todo, cibermedios y portales).

Dentro de los medios, se distinguen dos grupos a los que el gabinete de comunicación dirige las informaciones de la entidad: los de información general y los especializados. En España, en este segundo grupo cabe citar en el ámbito de los suplementos de prensa a Tercer Milenio, promovido en 1993 por el Grupo Heraldo de Aragón y que se entrega los martes con el diario; en el de las revistas a Quo, de periodicidad mensual en circulación desde 1993; programas de radio semanales como A Hombros de Gigantes, en emisión desde 2007 en RNE; en televisión Órbita Laika, que acaba de iniciar su tercera temporada en La2 o en Internet Materia, espacio que alberga en la actualidad Elpais.com y que fue puesto en marcha en 2012 por los promotores de ciencia del diario Público.

\section{¿Cómo comunica un gabinete de comunicación de un agente de ejecu- ción del Sistema?}

La principal decisión que deberá adoptar el responsable del gabinete de comunicación tras optar por difundir el tema a los medios de comunicación es si es objeto de una nota de prensa o de una rueda de prensa. Ambos conceptos han sido definidos por Ramírez (1995: 93-97).

Una nota de prensa es una declaración escrita (en el caso de la prensa) o realizadas en soporte magnético (radio/TV) sobre una cuestión concreta de interés general que envían las fuentes informativas a los medios de difusión. Debe estar redactada conforme a criterios periodísticos y por tanto ser breve, concisa y clara; abordar temas interesantes y de actualidad; tener una presentación correcta; enviarse a todos los medios y tener en cuenta sus especificidades. Desde su origen en la segunda mitad del siglo XIX, cuando el modelo de agencia de prensa estaba en su apogeo y el de información pública se estaba desarrollando (Grunig \& Hunt, 2003), es de los instrumentos más utilizados por el gabinete de comunicación.

Una rueda de prensa es una reunión convocada por una fuente informativa para dar cuenta de una determinada información a los medios de comunicación. Hay cinco pautas que se deben seguir cuando se opta por esta opción: debe estar justificada, correctamente realizada, hay que vigilar la duración de la comparecencia y la lista de participantes, los comparecientes tiene que ser capaces tanto de "ir al grano" como de evitar elegantemente preguntas conflictivas y hay que tener en cuenta la especificidad de cada medio.

En ambas hay que tener presente, además, la identidad visual corporativa, entendida por Martín (1999) como "un sistema de signos, formas, colores... que transporta ideas, impresiones psicológicas y una alta capacidad de memorización acerca de la personalidad de una empresa o institución". Túñez y Costa (2014) inciden en que ésta "se establece a lo largo de la vida de una compañía y puede modificarse en cualquier momento, si bien dicha modificación exige un profundo proceso de reflexión y análisis".

La nota de prensa debe estar redactada por el responsable del gabinete en base a la información facilitada por el personal investigador. Dicha información puede ser desde la memoria 
final de un proyecto de investigación al paper publicado en una revista científica. Es al responsable del gabinete a quien corresponde, en consonancia con el investigador, decidir el enfoque de la información e interpretarla y prepararla para su difusión a medios.

La extensión máxima de una nota de prensa sobre I+D+i, a nuestro parecer, debe ser de entre una y tres páginas. Su estructura puede incluir un antetítulo, un titular informativo, un par de despieces/destacados y el propio cuerpo de la nota. Se puede valorar la incorporación de citas de los investigadores para dar mayor dinamismo al texto.

Tienen que facilitarse en la nota de prensa los datos de contacto del responsable del gabinete, pero nunca directamente los del investigador. Es al gabinete al que corresponde gestionar las peticiones de los medios tras el envío de la información y distribuir los tiempos para atenderlos en base tanto a las necesidades del periodista como a la disponibilidad del investigador, a quien tiene que avisar previamente de que se le puede requerir para entrevistas en medios, por lo que se confirmará previamente con él la disponibilidad durante el día de difusión de la información.

La nota de prensa, redactada en estilo periodístico, debe llevar el logotipo de la entidad y tanto el tipo de letra como los colores corporativos de algunas letras (por ejemplo, las del titular) deben ser los que consten en el manual de identidad corporativa.

Su envío a los medios, una vez acordada la fecha con el personal investigador, se hará desde el gabinete de comunicación, preferentemente en horario de mañana. Puede acompañarse de fotografías o material multimedia que sirva para ilustrar la información a la que hace referencia.

El contenido de la nota de prensa puede emplearse posteriormente para difundir el tema en otros soportes, como la página web institucional o las redes sociales.

En cuanto a la rueda de prensa, el responsable del gabinete tendrá que preparar la convocatoria, que enviará a los medios con la antelación suficiente y con la información básica para llamar su atención y que acudan al acto. Se coordinará con el personal investigador a intervenir y decidirán el número de personas que harán declaraciones, así como el orden de intervenciones. También, el lugar en el que tendrá la rueda de prensa -es recomendable aprovechar la ocasión para reforzar la identidad visual corporativa, por ejemplo, con un panel tras los intervinientes con el logotipo de la entidad-. Se preparará una nota de prensa, más amplia, para entregar a los medios que acudan y para enviar posteriormente al resto.

Desde el gabinete se hará un seguimiento de lo que han recogido los medios de comunicación tras ambas acciones. Es importante que el responsable del gabinete haga saber y traslade al personal investigador que durante la rueda de prensa se deben aceptar preguntas y que por lo general los medios no publican la nota de prensa tal cual, sino que en las redacciones se prepara de nuevo la información. Esa labor de seguimiento derivará en el correspondiente dossier de medios o clipping de prensa para conocer la repercusión que ha tenido la información. Esas acciones, tradicionales, perviven y son la razón de ser de los gabinetes de comunicación de I+D+i. No obstante, tal y como afirma Pavlic (2005), "las innovaciones mediáticas están transformando el periodismo tradicional y tienen sus repercusiones en el periodismo de empresas e instituciones". 
En concreto, Internet ha transformado sustancialmente el universo de las comunicaciones (Adams, 2010: 67-82). Se ha convertido para las empresas e instituciones en una plataforma para todas sus comunicaciones de difusión, detección de necesidades de clientes y medios y de diálogo personal y colectiva, así como de interactividad, enlaces y navegación por todo lo que se necesite saber de la entidad (González, 2008).

Dentro de Internet, los agentes del Sistema de I+D+i disponen de dos grandes espacios para comunicar a su público: las webs institucionales y las cuentas oficiales en redes sociales.

Las webs de empresas e instituciones abren la opción a ofrecer permanentemente fotografías, organigramas, vídeos y documentos sonoros (Gallego, 2010), de interés para los medios y para el público en general (Küng et al. 2008). En ellas se enmarca el periodismo multimedia, entendido como una convergencia de informaciones procedentes de múltiples fuentes y distribuida, a su vez, en diversos medios integrados en grupos o en solitario para cubrir las necesidades de los usuarios, sea en su dimensión personal o en su dimensión social (Cebrián, 2010). La información que genera la entidad y que difunde a los medios a través de nota de prensa debe incluirse también en la página web institucional, espacio donde puede enriquecerla con material audiovisual y gráfico a disposición tanto de usuarios como de periodistas.

En cuanto a las redes sociales, también ahí debe darse cuenta de lo que se comunica a los medios, si bien se trata de un canal que permite contar otro tipo de información, de menor envergadura o que puede no ser susceptible de difusión mediática, y en un tono más informal que el empleado en la nota de prensa.

\section{¿Por qué comunica un gabinete de comunicación de un agente de ejecu- ción del Sistema?}

Hay numerosas razones que justifican que un agente de ejecución de I+D+i incorpore en su organigrama un gabinete de comunicación para encargarse, entre otras acciones, de sus relaciones con los medios de comunicación.

Tigeras y Ferrando (2013: 87-88) consideran que la comunicación de la ciencia a la sociedad debe hacerse para rendir cuentas sobre el gasto del presupuesto público destinado a la ciencia y tecnología, obtener retornos económicos para la investigación, renovar recursos humanos, garantizar el acceso de los ciudadanos a las fuentes del conocimiento, incrementar el conocimiento público sobre la investigación con fines diversos y favorecer una cultura democrática.

Fernández (2013. 19) también incide en que "hay infinitivas razones para divulgar la ciencia: el científico debe trasladar el entusiasmo por su trabajo, servir a los intereses de la comunidad científica, incrementar el interés ciudadano por la ciencia y, en definitiva, mejorar el conocimiento de la ciudadanía y los procesos democráticos, ya que una sociedad bien informada es una sociedad libre".

Hay, además, una necesidad que atender, como han puesto de relieve las ocho ediciones de la Encuesta de Percepción Social de la Ciencia y la Tecnología (FECYT: 2016): en algo más de una década el interés de la sociedad por la I+D+i ha pasado del 6,9\% (2004) al 16,3\% (2016).

Alcanzando una presencia mediática, periódica y justificada, los agentes dan visibilidad a su 
cometido y se pone en valor el trabajo del personal investigador. Al mismo tiempo, se contribuye a dar calidad y nivel a los contenidos periodísticos de los medios de comunicación, enriqueciendo su oferta informativa acorde a la sociedad del siglo XXI.

\section{Discusión}

Tanto por su importancia como por el tipo de informaciones que generan, es recomendable que los agentes de ejecución del Sistema Español de Ciencia, Tecnología e Innovación dejen en manos de profesionales de la comunicación sus relaciones con los medios a través de la incorporación en su organigrama de gabinetes de comunicación.

Éstos deben garantizar un flujo estable de información científica y tecnológica a los medios y una visibilidad periódica de la entidad para la que desarrollan su labor, pero tienen que evitar la sobresaturación informativa. Ciencia y tecnología en medios sí, claro, pero dosificada, justificada y amparada en rigor científico y criterios periodísticos.

Los gabinetes de comunicación de los agentes de ejecución del Sistema Español de Ciencia, Tecnología e Innovación, especialmente los públicos, deben ser una fuente compartida, de todos y para todos los medios, sin exclusiones. Tan importante es y debe ser que la ciencia y la tecnología que se realiza en una entidad abra un telediario nacional en horario de máxima audiencia como que se publique en un periódico local. En todos los medios hay público objetivo, un nicho de mercado, que tiene el derecho de recibir información científica y tecnológica. Y los gabinetes de comunicación de los agentes de ejecución del Sistema Español de Ciencia, Tecnología e Innovación contribuyen con su labor a garantizar ese derecho.

\section{Conclusiones}

Se puede conseguir presencia en los medios sin un gabinete de comunicación (Estévez, 2014), si bien para que los medios de comunicación divulguen la I+D+i es fundamental la consonancia de los siguientes factores: el compromiso de la comunidad científica, la labor de los Departamentos de Comunicación y la especialización de los periodistas de los medios (Bellón, 2016: 41).

En la actualidad, los gabinetes se ocupan tanto de las relaciones informativas con los medios como de las relaciones institucionales, la organización de eventos, las relaciones con la sociedad en general, la comunicación interna, etc (Almansa, 2011: 7-8). El presente artículo se ha centrado únicamente en las relaciones informativas por considerar que son su principal razón de ser.

Parafraseando a Ramírez (1995: 19) "la comunicación ha servido y sirve para que personas e instituciones que trabajan con seriedad vean sus esfuerzos compensados en los medios de difusión. Pero no admite abusos". La comunicación científica, tampoco. Debe estar al servicio de la sociedad, al servicio del ciudadano, del público no especializado, que busca en un medio de comunicación un canal con el que alfabetizarse e incrementar su cultura científica.

Hay una amplia tipología de gabinetes de comunicación, que se ha ido incrementando en las últimas décadas. Así, se ha llegado también a la implantación progresiva, en los agentes de ejecución del Sistema Español de Ciencia, Tecnología e Innovación, de un modelo especializado en I+D+i. 
Su día a día y sus funciones son similares a las de cualquier gabinete: elaborar y difundir a medios notas de prensa, organizar y convocar ruedas de prensa, gestionar entrevistas, elaborar el clipping de prensa... Es decir, las habituales líneas de actuación, las más tradicionales.

No obstante, los especializados en I+D+i trabajan con un activo intangible de primer nivel: el conocimiento científico y tecnológico. Su principal cometido es que éste entre en la agenda de los medios de comunicación, lo que reviste ciertas complicaciones: falta de espacio, competencia de otros temas y dificultad para comunicar con claridad, rigor e interés para el público no especializado ese conocimiento. Su cometido, también, es implicar y contar con la colaboración del personal investigador para hacer llegar ese conocimiento a los medios.

Según García (2009: 11) "los gabinetes de comunicación, como emisores privilegiados durante los últimos años, deben buscar nuevas fórmulas para comunicar y recibir información emitida sobre la entidad". En el ámbito de la I+D+i, los retos a los que se enfrenta y se lleva enfrentando este tipo de gabinetes son, cuanto menos, claves para conseguir una alfabetización científica y tecnológica de la sociedad a través de los medios. De que el responsable del gabinete sepa navegar entre los dos mundos que conecta, el científico y el periodístico, y de que sea capaz de conjugar el valor científico y tecnológico de un hecho con los valores noticia depende, en parte, que la comunicación científica y tecnológica ocupe el lugar que merece en el panorama mediático.

\section{Referencias bibliográficas}

- AA.VV. (2002). Comunicación empresarial. Barcelona: Gestión 2000.

- $\quad$ Adams, T. (2010). Bases de la nanotecnología. Comunicación de choque. Lima: Universidad de San Martín de Porres. Fondo Editorial.

- Almansa Martínez, A. (2011). Del gabinete de prensa al gabinete de comunicación. Sevilla: Comunicación Social Ediciones y Publicaciones.

- Almansa Martínez, A. (2006). Gabinetes de comunicación. Estudio sobre su presencia en las organizaciones. Anàlisi, 34, 223-237. Recuperado el 10 de octubre de 2017 de http:// www.raco.cat/index.php/Analisi/article/view/55454

- Almansa Martínez, A. (2004). Historia de los gabinetes de comunicación en España. Revista Historia y Comunicación Social, 9, 5-12. Recuperado el 10 de octubre de 2017 de http:// revistas.ucm.es/index.php/HICS/article/view/HICS0404110005A

- Andrade, H. (2004). Comunicación organizacional interna: proceso, disciplina y técnica. Coruña: Netbiblio.

- Asociación Española de Normalización y Certificación (2008). Normas UNE. Gestión de la I+D+i. Terminología y definiciones de la actividad de I+D+i (UNE 166000).

- Camacho Markina, l. (2001). Funciones de los gabinetes de prensa en la administración local. Revista Latina de Comunicación Social, 4, 44. Recuperado el 10 de octubre de 2017 de

- http://www.revistalatinacs.org/2001/latina44septiembre/4403camacho.htm

- Bell, D. (1973). The coming of post-industrial society. Nueva York: Basic Books. Bellón Rodríguez, A. (2016). La radio pública estatal en España como medio de empoderamiento de las personas a través de la alfabetización científica: A hombros de gigantes y Entre probetas. Communication Papers, 5 -10, 39-57. Recuperado el 10 de octubre de 2017 de http:// 


\section{ojs.udg.edu/index.php/CommunicationPapers/article/view/BELLON}

- Capriotti, P. (1999). Comunicación corporativa. Una estrategia de éxito a corto plazo. Reporte C\&D - Capacitación y Desarrollo, 13, 30-33.

- Carrascosa, JL. (1992). ComunicaACCION. Una comunicación eficaz para el éxito de los negocios. Madrid: Ciencias de la Dirección.

- Cebrián Herreros, M. (2012). Periodismo empresarial e institucional. Sevilla: Comunicación Social Ediciones y Publicaciones.

- Cebrián Herreros, M. (2010). Desarrollos del periodismo en Internet. Sevilla: Comunicación Social Ediciones y Publicaciones.

- Costa, C. (2011). El gabinete de comunicación del hospital. Propuesta teórica y

- acercamiento a la realidad de los departamentos de comunicación de los hospitales públicos de Galicia. DOXA, 14, 175-197. Recuperado el 10 de octubre de 2017 de https://recyt. fecyt.es/index.php/doxacom/article/view/35983

- Dirección Xeral de Investigación e Desenvolvemento (2003). Guía básica de gestión de proyectos de I+D+i. Santiago de Compostela: Dirección Xeral de Investigación e Desenvolvemento.

- Elías, C. (2008). Fundamentos de periodismo científico y divulgación mediática. Madrid: Alianza Editorial.

- Elías, C. (1998). Más allá de la comunicación interna: la intracomunicación. Barcelona: Gestión 2000.

- Estévez, L. (2014). Cómo conseguir presencia en los medios sin un gabinete de prensa. Barcelona: UOC.

- Fernández del Moral, J. \& Esteve Ramírez, F. (1993). Fundamentos de información periodística especializada. Madrid: Editorial Síntesis.

- Fernández Asenjo G. \& Torre Alfaro N. (2009). Gabinetes de comunicación en mínimos. Madrid: Fragua.

- Fernández de Lis, P. (2013). Ciencia y periodismo en la red. En El científico ante los medios de comunicación. Retos y herramientas para una cooperación fructífera (15-21). Barcelona: Fundación Dr. Antonio Esteve.

- Fundación Española para la Ciencia y la Tecnología (2016). VIII Encuesta de Percepción Social de la Ciencia y la Tecnología. FECYT: Madrid. Disponible en https://www.fecyt.es/ es/publicacion/percepcion-social-de-la-ciencia-y-la-tecnologia-en-espana-2016

- Fundación Española para la Ciencia y la Tecnología (2016). La Ciencia es Noticia. FECYT: Madrid. Disponible en https://www.fecyt.es/es/publicacion/anuario-sinc-la-ciencia-es-noticia-2017

- Fontcuberta, M. (2011). La noticia. Pistas para percibir el mundo. $1^{\circ}$ edición revisada y ampliada. Barcelona: Paidós.

- $\quad$ Galtung J \& Ruge MH. (1973). Structuring and selecting news. En Cohen \& Young, The manufacture of news: Social Problems, Deviance and the mass media. Londres, Constable.

- Gallego, Jl. (2010). Podcasting: nuevos modelos de distribución para los contenidos sonoros. Barcelona: UOC.

- García Orosa, B. (2009). Gabinetes de comunicación online. Claves para generar información corporativa en la red. Sevilla: Comunicación Social Ediciones y Publicaciones.

- González López, O.R. (2008). Manual imprescindible de Internet para la empresa. Madrid: Anaya Multimedia.

- Grunig J.E \& Hunt, T. (2003). Dirección de relaciones públicas. Barcelona: Gestión 2000. 
- $\quad$ Küng ,L., Picard, RG \& Towse, R. (2008). The Internet and the Mass Media. Londres: SAGE Publications.

- La Colic, Y.F. (2006). La science de l'information. París: PUF.

- Martínez Albertos, JL. (1983). Curso general de redacción periodística. Barcelona: Mitre.

- Martín Martín, F. (1999). Comunicación empresarial e institucional. Madrid: Editorial Universitas.

- Mateos Martín, C. (2003). Descripción de la estructura funcional del gabinete de prensa político en los entornos informativos locales. Revista Latina de Comunicación Social, 6, 54. Recuperado el 10 de octubre de 2017 de http://www.revistalatinacs.org/20035414mateos.htm

- Moreno Castro, C. (Ed.) (2009). Comunicar los riesgos. Ciencia y tecnología en la sociedad de la información. Madrid: Biblioteca Nueva-Organización de Estados Iberoamericanos.

- Pavlic, J. (2005). El periodismo y los nuevos medios de comunicación. Barcelona: Paidós.

- Ramírez, T. (1995). Gabinetes de Comunicación. Funciones, disfunciones e incidencia. Barcelona: Bosch Comunicación.

- Rositi, T. (1980). Historia y teoría de la cultura de masas. Barcelona: Gustavo Gili.

- Showmaker, P. (1991). Comunication Concepts 3: Gatekeeping. Londres. VER

- Túñez M. \& Costa C. (2014). Comunicación corporativa: claves y escenarios. Barcelona: UOC.

- Túñez, M. (1999). Producir noticias. Cómo se fabrica la realidad periodística. Santiago de Compostela: Tórculo.

- Tigeras P \& Ferrando L. (2013). Cultura científica, cultura democrática. En El científico ante los medios de comunicación: retos y herramientas para una comunicación fructífera, (8595). Barcelona: Fundación Dr. Antonio Esteve.

\section{Otras referencias}

Ley 14/2011, de 1 de junio, de la ciencia, la tecnología y la innovación (BOE 02/06/2011). Disponible en https://www.boe.es/boe/dias/2011/06/02/pdfs/BOE-A-2011-9617.pdf

\section{CURRICULUM VITAE. ANA BELLÓN RODRÍGUEZ}

Doctora y licenciada en Periodismo por la Universidad de Santiago de Compostela. Máster en Edición Periodística por la Universidad de A Coruña. Responsable de Comunicación del Consejo Superior de Investigaciones Científicas en Galicia. Profesora asociada en la Facultad de Ciencias de la Comunicación de la Universidad de Santiago de Compostela. Miembro del grupo de investigación Novos Medios. Sus líneas de investigación son la prensa gratuita y el periodismo científico. 

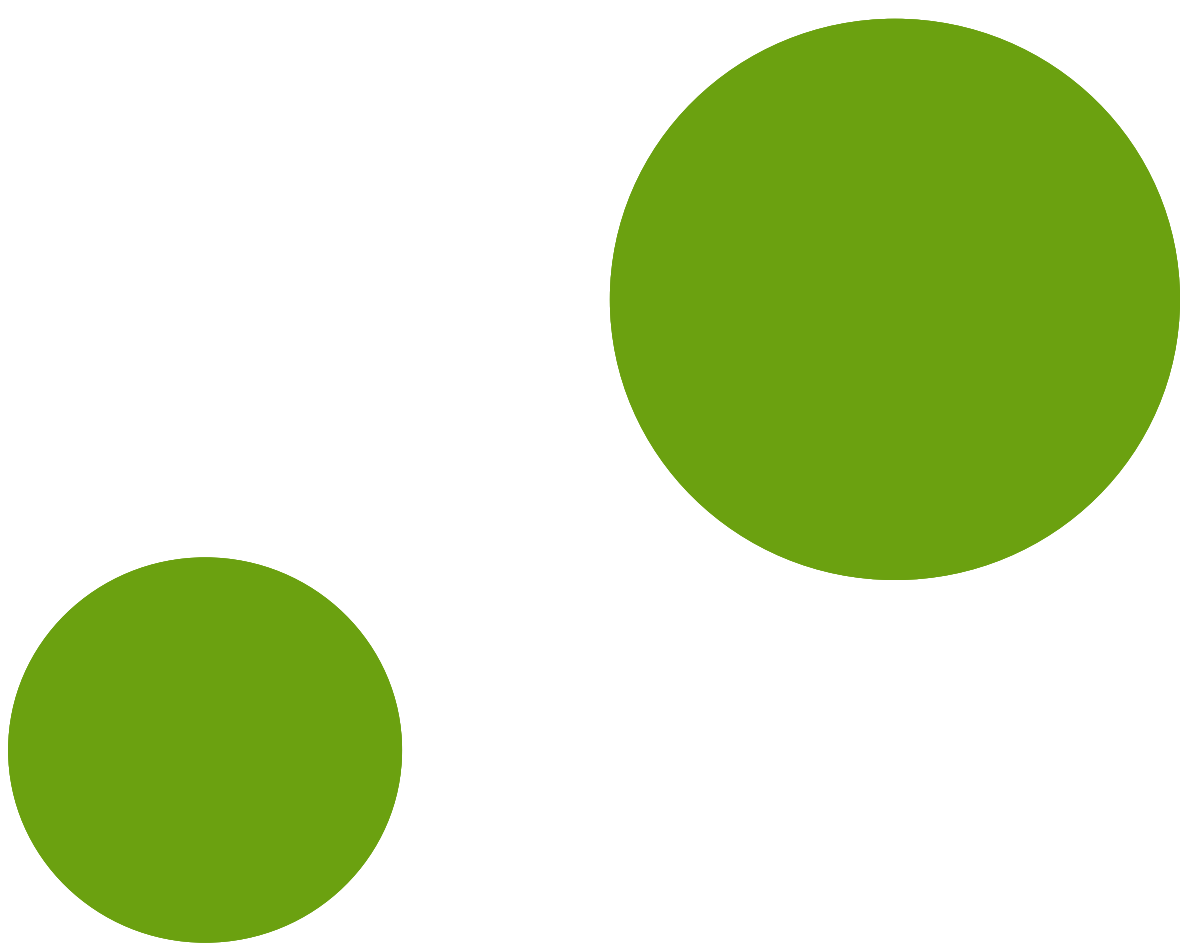
